\title{
Enrichment Analysis of hsa-miR-320a most Related Pathways in Breast Cancer
}

\author{
Sahar Janghorban ${ }^{1,}$, Pardis Saadatmand ${ }^{2}$, Mansoureh Azadeh ${ }^{2,3}$, \\ Kamran Ghaedi ${ }^{4}$, Morteza Asgari ${ }^{5}$ \\ ${ }^{1}$ Division of cellular and Molecular Biology, Department of Biology, Nour Danesh \\ Institute of Higher Education, Meymeh, Isfahan, Iran \\ ${ }^{2}$ Zistfanavary Novin, Biotechnology Institute, Isfahan, Iran \\ ${ }^{3}$ Department of Biochemistry, Faculty of Basic Science, Najafabad Branch, Islamic \\ Azad University, Najafabad, Isfahan, Iran \\ ${ }^{4}$ Division of Cellular and Molecular Biology, Department of Biology, Faculty of \\ Science, University of Isfahan. Isfahan, Iran \\ ${ }^{5}$ Department of Biology, faculty of Basic Science, Nour Danesh Institute of Higher \\ Education, Meymeh, Isfahan, Iran \\ ${ }^{*}$ Corresponding author: Sahar Janghorban, Division of cellular and Molecular \\ Biology, Department of Biology, Nour danesh Institute of Higher Education, Meymeh,
}

DOI: $10.21859 / \mathrm{mci}$-supp-24

\section{Keywords:}

Breast Cancer

Signaling Pathway

microRNA

hsa-miR-320a

\begin{abstract}
Introduction Breast cancer is the most common cancer and the second leading cause of death among women. The study of this disease in countries, the problems and costs of treatment for families and individuals is a factor for identification of biomarkers. MicroRNAs are a group of small non-coding RNAs that include 18-25 nucleotide length and involve in regulation processes by translation inhibition. MicroRNAs usually do this by binding to complementary sequences located in 3'UTR of target mRNA. MicroRNA as a biomarker regulates and controls the level of mRNA expression and exerts its effect as oncomiR or tumor suppressor.

Materials and Methods: By considering the role of hsa-miR-320a in breast cancer, for obtaining predicted targets of hsa-miR-320a, MIMAT ID of active arm was picked from miRBase database and inserted in miRWalk2.0 database. Finally, for obtaining the most related pathways of hsa-miR-320a, the entrez IDs of predicted targets were inserted into DAVID database.

Results: Our data manifested KEGG signaling pathways "GLIOMA" and "pathway in cancer" and "MAPK signaling pathway" as the most statistical relevant pathways with hsamiR-320a targetome.

Conclusions: Information from all of above signaling pathways indicates that microRNA targets the GRB2 gene and prevents the activation of the sos, Ras, Raf, MEK genes. Also targeting the ERK gene prevents cell growth and proliferation. According to these studies, it is predicted that hsa-miR-320a acts as a tumor suppressor microRNA that prevents the activation of a number of genes that are involved in the process of "cell growth" and "proliferation" and may be in prognosis of breast cancer by altering some of the signaling vital ones.
\end{abstract}

